\title{
Plant Disorder Identification System by Implementing Image Classification Technique
}

\author{
Madhav Singh Solanki
}

\begin{abstract}
Crop production in the sector of agriculture performs an important part to complete daily food needs. The failure of food is largely linked to infected plants, crops which decreases the production rate reflectively. Plant illnesses are not yet investigated in a premature stage to define. The primary difficulty is the lessening in the use of pesticides in farming and the rise in manufacturing performance and volume. They have suggested an improved clustering method for predicting the region of the affected leaf in this paper. The intent of this paper is to use portable technological development to promote understanding of crop organisms around us. A color-based segmentation system is described to separate and classify the affected area of the plant. Experimental analyzes of temporal complexity and the area of affected areas in sample pictures have been supported out, the picture processing technique which can detect illnesses of the plants. Disease identification includes measures such as picture creation, pre-processing, division of the picture, removal of features and ranking, initiative detects crop illnesses and provides alternatives for illness recovery. It indicates the proportion of the impacted portion of the leaf of a plant.
\end{abstract}

Keywords - Disease Detection, Production rate, K-means clustering, Voice navigation, Infection region.

\section{INTRODUCTION}

India is a prominent country for agriculture, which implies that most individuals are involved in farming. In the financial industries, the agricultural industry plays an important part. Variant fungal and bacterium illnesses infect most crops. Because of the human population accelerating trend, the climate also results in plant disease. Sustainable development is challenged primarily by reducing pest control use, environmental protection and performance improvements. The use of insecticides may be reduced by precision, accuracy and premature detection. Farmers are making considerable efforts to pick the finest crop varieties and also ensure the right atmosphere for plant development, although plants lead to plant infection with a number of illnesses. In agriculture, it really is essential in the initial stages to find the crop disorders that assist the farmer to lessen harm, decrease the cost of manufacturing and increase revenue. Several other occasions the natural lens itself is not so efficient in identifying the right illness. On the basis of the recommendations of scientists, landowners have taken remedial measures to heal crop conditions and then used bare vision study by specialists with specimens of impacted crops or by specialists used only to attend the farm. In this technique, reliable experts are very hard to find and the alternative doesn't function well for big areas. This

Revised Manuscript Received on 14 September, 2019.

Madhav Singh Solanki, Department of Computer Science and Engineering, Sanskriti University, Uttar Pradesh,India. (E-mail: sanpubip@gmail.com) approach is also costly, as it calls for ongoing expert surveillance. Close surveillance scheme for illnesses affecting the manufacturing and post-harvest lives is obligatory in order to handle vegetable leaves. The notion of information mining techniques picture rescue helps in: recognition of trapped leaves and stems ii) measurement of area form of the region iv) determination of the color of the region v) and impact of plant volume and structure.

\section{RELATED WORK}

In this sector, many scientists have carried out studies. A paper [1] discusses the edge tracking method to segment the picture, and the HPCCDD tool for image analysis and diagnosis of illnesses has at last been suggested. The objective of that study was to discover the illnesses in the place of pine leaves through picture production and evaluate the original pictures through the RGB pixel calculation and to locate the area of the leaves by the method and performance of Sobel and Canny Edge. Image segmentation methods defined the computer template scheme for illness recognition by using picture growth. Another paper [2] suggested a prototype system software for recognizing plant sickness by implementing image segmentation methods and algorithms on that captured image.

Another published paper [3] et al. discloses about the researched techniques for processing an image and to implement this cucumber powdery mildew there studies as sample data. Another research was done by [4] 2010; deliberated about the regularization and removal technique, giving more precision than other tracking characteristics.

Another paper [5] in same year deliberated about three techniques for disease detection on a leaf. The first method disclosed about recognizing a part of leaf employing by clustering of image captures. The second method discloses the treatment of the injured part of the leaf by means of texture analysis.

A paper in 2015 [6] suggested an approach to avoid yield from dying by detection of disease occurring in the crop. In the same year [7] discloses about a technique based on automated technology for recognizing diseased leaves by implementing the grayscale algorithm using color data of leaves and a paper [8] discusses artificial neural network which is trained to differentiate between normal and diseased samples.

\section{PROPOSED SYSTEM}

The initiative consists of detecting illnesses of plants and providing alternatives for leaf recovery. The intended to

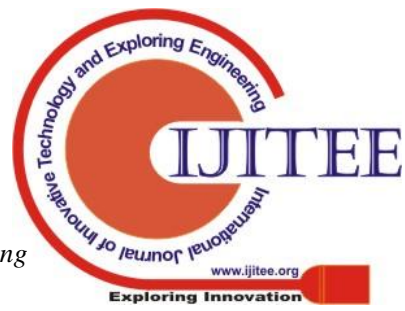


build the venture with a speech tracking scheme to make it easy for an individual with little computer knowledge to use. In the suggested framework provide a remedy for the recovery from plant illnesses as well as a picture handling method showing the impacted portion of the leaves. Only the disease from affecting the blade can be identified by the current scheme. Specimens of 75 pictures comprising various crop diseases like as Alternaria, Anthracnose, Cercospora Leaf and Sanitary Leaves are gathered and tested from those 75 pictures. The test screen shows the identification of crop illness using a color-based template.

Proposed $\mathrm{k}$ means clustering protocol comprises of several steps, image accession, image preprocessing, and detection of special attributes form captured the image and natural network-based categorizing. The first method consists of gathering information from the database. It requires the picture as an entry to process further. They've been using the most far and wide-known picture fields to use any formats as.bmp,.jpg,.gif The pictures can comprise as noise dust, spores and water places when obtained from the true domain. Preprocessing data is intended to remove noise in the captured picture so that the pixel attributes can be adjusted. It improves the picture performance. The final stage in the suggested technique is image standardization. The segmented pictures are divided into distinct industries using the clustering method Otsu and K-mean. The Red, Blue, Green (RGB) color system is converted into a Lab color template until the image is clustered. The emergence of Lab color is that the segmented pictures are readily clustered.

"K-means Clustering Algorithm"

The classifier divides the objects into $\mathrm{k}$ resemblance communities. Euclidean distance is used as a metric to calculate this resemblance. The algorithm operates like this: the start $\mathrm{k}$ values, called implies. The initialize them then classify each pair in the nearest average and check the average co-ordinates which constitute the scores of the products so far. Replication of this method is done for a certain amount of iterations and finally, the nodes are computed.

a) Load the pictures of feedback.

b) Switch the RGB picture to a color space of $\mathrm{L}^{*} \mathrm{a} * \mathrm{~b}$.

c) RGB images are primary (red, green, blue) color combinations.

d) The pixel counting method of the red, blue, green image character is widely used for agriculture.

e) $\mathrm{L} * \mathrm{a} * \mathrm{~b} *$ room is a radiation sheet ' $\mathrm{L} *$ ' which shows a pixel image along the red-green border and a colorations sheet ' $b *$ ' showing where the image lies along the blueyellow border. The background sheet is a chromaticity matrix. The data for all colors is available in the stages ' $a *$ ' and ' $b$ *. '

f) Clustering colors with the technique k-mean.

g) The Euclidean range is described as following between two items:

$$
\operatorname{Dis}_{(a, b)}=\sqrt{\sum_{i}\left(x_{i}-y_{i}\right)^{2}}
$$

h) Each image is clustered according to its calculated cluster-center version.
The spotting of features is essential for a graceful prediction of the affected area. Here is the spotting of the form and textural function. The form orientated removal function is calculated such as the area, distance of the core, eccentricity, solidity, and perimeter. Likewise, textures such as comparison, correlation, pother, homogeneity, and average are designed for the extracted characteristics.

Hence, the designed system architecture is shown in Figure 1. The first method is just to pick the picture. The leaf must be identified even if it is not influenced by the picture preprocessing method. Then the user may segment the picture and identify the title of the disorder. This initiative offers a response to plant illnesses and analyzes the general proportion of the leaves concerned and the adjacent area.

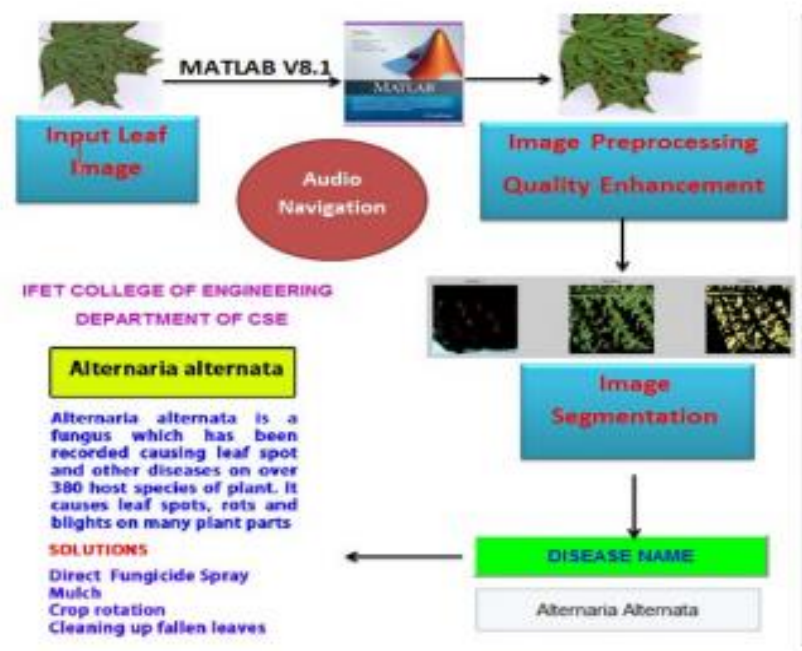

Figure 1 System design

Table 1 depicts the measurement of time complexity and region detection of the infection area.

Table 1 measurement of the infected area

\begin{tabular}{|l|l|l|l|}
\hline Types of diseases & $\begin{array}{l}\text { No. of } \\
\text { images }\end{array}$ & $\begin{array}{l}\text { Clustering time } \\
\text { (s) }\end{array}$ & $\begin{array}{l}\text { Area of } \\
\text { infected } \\
\text { region (\%) }\end{array}$ \\
\hline $\begin{array}{l}\text { Alternaria } \\
\text { Alternata }\end{array}$ & 22 & Below 20 S & 15.0062 \\
\hline Anthracnose & 23 & Below 20 S & 15.0915 \\
\hline Bacterial Blight & 7 & Below 20 S & 13.0093 \\
\hline $\begin{array}{l}\text { Cercospora leaf } \\
\text { spot }\end{array}$ & 9 & Below 20 S & 18.2951 \\
\hline
\end{tabular}

\section{RESULT}

Image is captured from the real field and it may comprise of dirt powder, spores and even water spots which act as noise in the process of image preprocessing. Image preprocessing is employed in this proposed framework to remove the aforementioned noise data in the image as shown in figure 2 . 


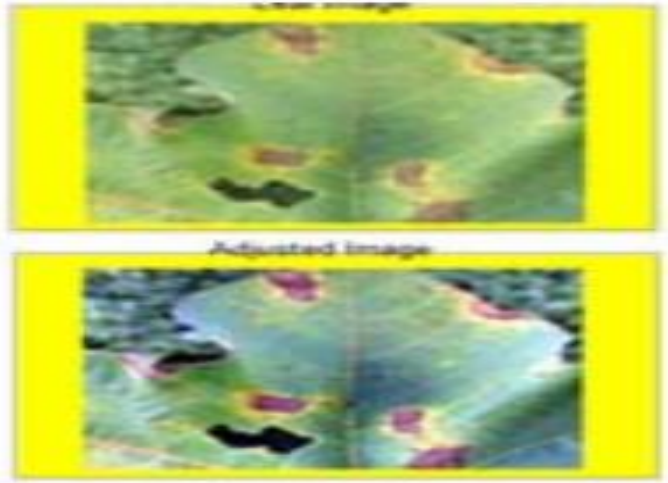

Figure 2 Image preprocessing

Otsu's image classifier is employed for clustering threshold of the image. Figure 3 shows the Otsu's classification on the leaf image.

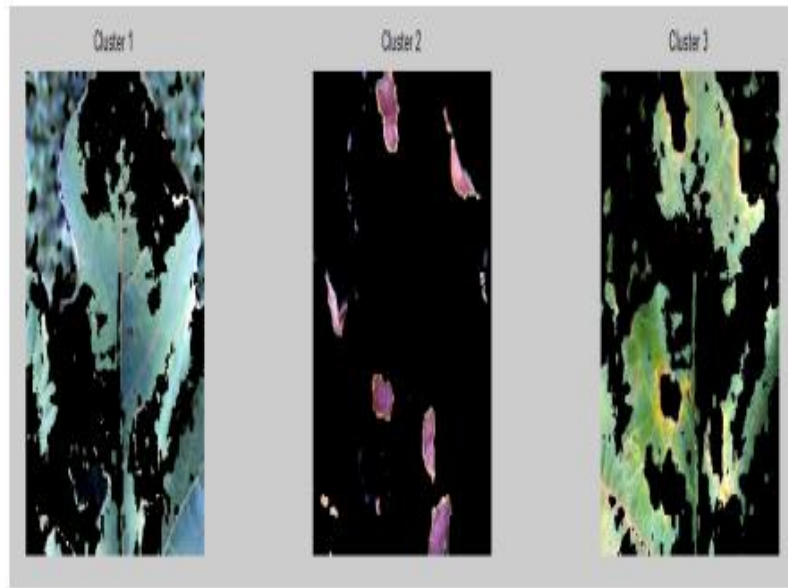

Figure 3 Otsu's classification

\section{1. "Torula alternate"}

It is a disease that affects the leaf places that have crop populations over 380 . The leaf locations, reds, bite and other plants may also be predisposed.

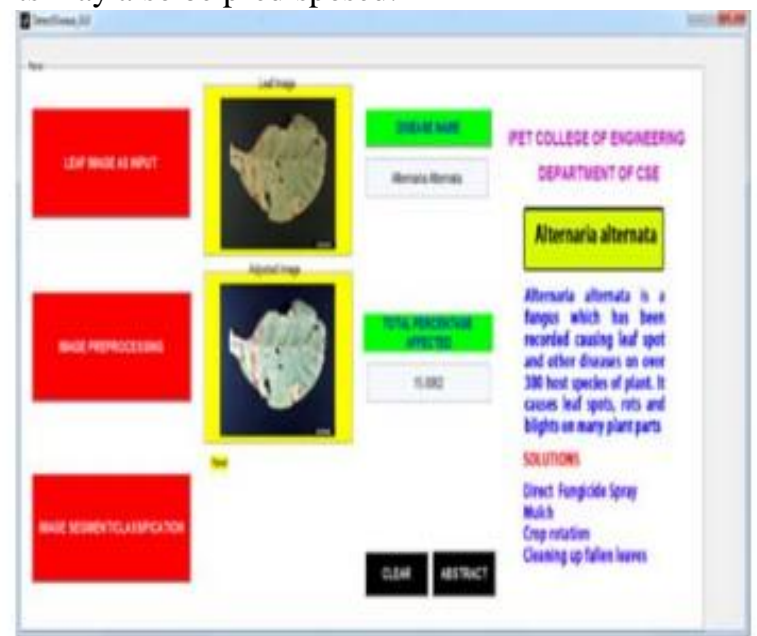

Figure 4 Alternaria rot fungus

\section{2. "Xanthomonas oryzae PV. Oryzae"}

Bacterial Blight is described as water-sprinkled by tiny, pure yellow patches or marks. The tumors then grow into the deceased places. The whole duration of the leaf can stretch.

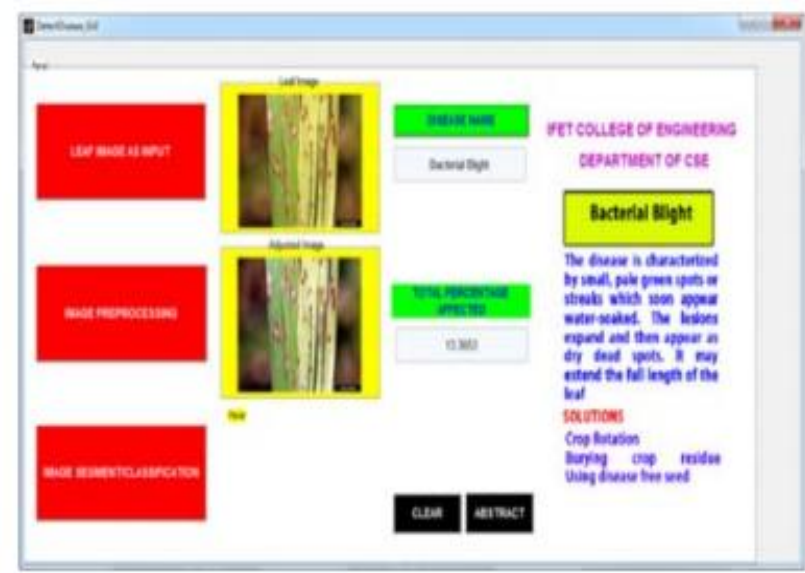

Figure 5 Xanthomonas oryzae PV. Oryzae

\section{CONCLUSION}

Accurate recognition and classification of plant illness are essential for enhancing the cultivation of the crop. And this is employed by image processing techniques. This paper has proposed an enhanced way of image segmentation of plant and detection of respective disease. The scheme proposed indicates the sophisticated method, which is a small computing effort can clearly promote an exact diagnosis of plant illnesses. Data mining methods there incorporated in the agricultural sector. This initiative carries out an advanced concept to recognize plants impacted and to provide the crop sector with remedial interventions. Feature removal method enables the damaged leaves to be extracted and crop illnesses to be classified. Built-in speech tracking scheme enables the operator to control the whole method..

\section{REFERENCES}

1. Bankar, Shital, et al. "Plant disease detection techniques using canny edge detection \& color histogram in image processing." International Jthenal of Computer Science and Information Technologies 5.2 (2014): 1165-1168.

2. Santanu Phadikar \& Jaya Sil [2008] Rice Disease Identification Using Pattern Recognition Techniques,Proceedings Of 11th International Conference On Computer And Information Technology, 25-27

3. Geng Ying, Li Miao, Yuan Yuan \&Hu Zelin[2008] A Study on the Method of Image PreProcessing for Recognition of Crop Diseases, International Conference on Advanced Computer Control, 2008 IEEE.

4. Ajay A. Gurjar, Viraj A. Gulhane," Disease Detection on Cotton Leaves by Eigenfeature Regularization and Extraction Technique", International Jthenal of Electronics, Communication \& Soft Computing Science and Engineering (IJECSCSE) Volume 1, Issue 1.

5. H. Al-Hiary, S. Bani-Ah Mad, M. Reyalat, M. Braik And Z. A Lrahamneh, Fast And Accurate Detection And Classification Of Plant Diseases, IJCA, 2011, 17(1), 3138, IEEE-2010.

6. PawanP.Warne, Dr.S.R. Ganorkar" Detection of Diseases on Cotton Leaves Using K-Mean Clustering Method”, International Research Jthenal of Engineering and Technology(IRJET) Volume: 02 Issue: 04 | July-2015, 425-431.

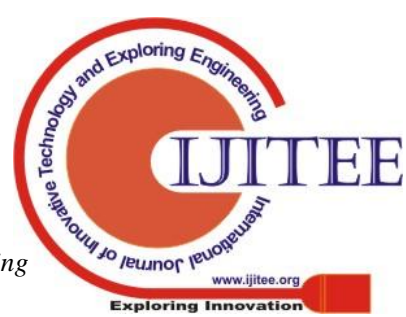


7. Daisy Shergill, AkashdeepRana, Harsimran Singh "Extraction of rice disease using image processing",International Jthenal Of Engineering Sciences \& Research technology,June, 2015,135- 143.

8. Malvika Ranjan1, Manasi Rajiv Theginwar,NehaJoshi,Prof.A.B. Ingole, detection and classification of leaf disease using artificial neural network,International Jthenal of Technical Research and Applications e-ISSN: 2320-8163, Volume 3, Issue 3 (May-June 2015), PP. 331-333

9. K. Padmavathi, and K. Thangadurai, "Implementation of RGB and Gray scale imagesin plant leaves disease detection -comparative study," Indian J. of Sci. and Tech., vol. 9, pp. 1- 6,Feb. 2016.

10. Dr.K.Thangadurai, K.Padmavathi, "ComputerVision image Enhancement For Plant LeavesDisease Detection", 2014 World Congress on Computing and Communication Technologies.

11. Kiran R. Gavhale, and U. Gawande, "An Overview of the Research on Plant Leaves International Jthenal of Pure and Applied Mathematics Special Issue 882 Disease detection using Image Processing Techniques," IOSR J. of Compu. Eng. (IOSRJCE), vol. 16, PP 10-16, Jan. 2014.

12. Y. Q. Xia, Y. Li, and C. Li, "Intelligent Diagnose System of Wheat Diseases Based on Android Phone," J. of Infor. \& Compu. Sci., vol. 12, pp. 6845-6852, Dec. 2015.

13. Thenjiang Huang, Qingsong Guan, JuhuaLuo, Jingcheng Zhang, Jinling Zhao, Dong Liang, Linsheng Huang, and Dongyan Zhang, "New Optimized Spectral Indices for Identifying and Monitoring Winter Wheat Diseases", IEEE jthenal of selected topics in applied earth observation and remote sensing, Vol. 7, No. 6, June 2014

14. Monica Jhuria, Ashwani Kumar, and RushikeshBorse, "Image Processing For SmartFarming: Detection Of Disease And Fruit Grading", Proceedings of the 2013 IEEE Second International Conference on Image Information Processing (ICIIP-2013)

15. Zulkifli Bin Husin, Abdul Hallis Bin Abdul Aziz, Ali Yeon Bin MdShakaffRohaniBinti S Mohamed Farook, "Feasibility Study on Plant Chili Disease Detection Using Image Processing Techniques", 2012 Third International Conference on IntelligentSystems Modelling and Simulation.

16. Mrunalini R. Badnakhe, Prashant R. Deshmukh,"Infected Leaf Analysis and Comparison by Otsu Threshold and kMeans Clustering", International Jthenal of Advanced Research in Computer Science and Software Engineering, Volume 2, Issue 3, March 2012.

17. Sharif, Muhammad, et al. "Detection and classification of citrus diseases in agriculture based on optimized theighted segmentation and feature selection." Computers and electronics in agriculture 150 (2018): 220-234

18. Yasmin, Ghazaala, Asit K. Das, and Arijit Ghosal. "A hierarchical stratagem for rice leaf disease distinction." 2017 International Conference on Intelligent Computing, Instrumentation and Control Technologies (ICICICT). IEEE, 2017.

19. Golhani, Kamlesh, et al. "A review of neural networks in plant disease detection using hyperspectral data." Information Processing in Agriculture 5.3 (2018): $354-$ 371.

20. Barbedo, Jayme GA. "Factors influencing the use of deep learning for plant disease recognition." Biosystems engineering 172 (2018): 84-91 